\title{
PREDICTION OF IMAGE DETAIL
}

\author{
D. Darian Muresan and Thomas W. Parks
}

\author{
School of Electrical Engineering, Cornell University \\ Ithaca, NY 14853 \\ darian,parks@ee.cornell.edu
}

\begin{abstract}
In the problem of image interpolation, most of the difficulties arise in areas around edges and sharp changes. Around edges, many interpolation methods tend to smooth and blur image detail. Fortunately, most of the signal information is often carried around edges and areas of sharp changes and can be used to predict these missing details from a sampled image. A method for adding image detail based on the cone of influence, the evolution of the wavelet coefficients across scales, is presented in this paper.
\end{abstract}

\section{INTRODUCTION}

In its most basic form, image interpolation generates a larger image from a smaller size image. Two different types of interpolation can be classified as enlargement and zooming.

In the enlargement case, the idea is to decompose an image into a collection of basis functions and then stretch those basis functions to enlarge the image. Think of it as projecting an image onto a large screen. In this category, some well known methods are pixel replication (the basis functions are assumed to be the standard piecewise constant functions), zero padding in the frequency domain (the basis functions are sines and/or cosines) and zero padding in the wavelet domain (the basis functions are the wavelets). The enlargement method works very well when the image resembles the basis functions. For example, if we had a checker board image, pixel replication would work perfectly. However, these methods may not work as well when used on real images. For example, zero padding in the frequency domain tends to introduce a lot of ringing around edges.

The second type of interpolation is what we would call zooming. In the zooming case, one would like to add in extra detail as the image is enlarged. Think of zooming as looking at an image through a microscope: the image becomes larger, but you also have more detail. This second type of interpolation requires an image model, in order to predict lost detail. In this category, there is a range of different interpolation techniques. Some well known techniques

This work was supported by NSF MIP9705349, TI and Kodak are linear interpolation (with the model that the image is locally linear) and cubic interpolation (with the model that the image is locally cubic). More recently interesting results have been obtained using wavelets in trying to model real images and in particular, to model smoothness found in real images $[1,2,4,6,7,8]$. By studying regularity measures of the image, details can be added to the image by constraining the image to the known (or assumed) regularity.

This paper is organized as follows. Section 2 is a review of previous work [1, 2, 4, 6, 7]. It also introduces some of the basic definitions and concepts which are required later on. Section 3 presents our main idea and approach of adding image detail based on the information of the wavelet coefficients at coarser scales. Section 5 presents some of our computational results and finally section 6 will conclude the paper with future work that needs to be done in this direction.

\section{REVIEW}

A promising new approach to prediction over scale using Hidden Markov Models (HMM) has been developed recently [2]. Here the HMM is trained using an image database. Once the parameters of the model are obtained, the prediction of the detail signal is made and combined with the measured coarse scale signal to reconstruct a cleaner image. In [2] the algorithm was used for denoising by trying to predict the correct values of the coefficients at the finest scale from the coefficients at the coarser scales. The idea is that the coarser scale coefficients are less affected by noise, while the detail coefficients contain most of the noise. The denoising idea can be easily extended to interpolation.

Other two methods of estimating detail based on information across scale are largely based on the work and observations made in $[6,7]$. First, let's introduce some definitions and notation.

The undecimated dyadic wavelet transform is computed by projecting a discrete signal $f$ onto a set of translations and dilation of a mother wavelet $\psi$ :

$$
\psi_{s, l}(n)=2^{-\frac{s}{2}} \psi\left(2^{-s} n-l\right)
$$


where the scale $s$ and the offset $l$ are integers. The wavelet transform coefficients are then $w_{s, l}=\left\langle f, \psi_{s, l}\right\rangle$. In the dyadic wavelet transform, each sample of $f$ has a cone of influence [3]. For completeness, we provide the definition here.

Definition 1 The cone of influence of $f\left(n_{0}\right)$ in the scalespace plane is the set of points $(s, l)$ such that $n_{0}$ is included in the support of $\psi_{s, l}$.

In $[6,7]$ the mathematical characterization of singularities with Lipschitz exponents is explained. Looking at the dyadic wavelet decomposition, the wavelet transform modulus maxima (the local maxima of the wavelet coefficients at a given scale) locates all the singularities of a function by following all the maxima lines when the scale goes to zero. Given a function $f$ defined on $[a, b]$ ( $a, b$ integers) and index $n_{0} \in$ $[a, b]$, if there exists a scale $s_{0}>0$ and a constant $C$ such that the modulus maxima of the wavelet coefficients belong to a cone defined by

$$
\left|l-n_{0}\right| \leq C 2^{s}
$$

then $f$ is uniformly Lipschitz [6]. Moreover, there exists constants $A$ and $\alpha$ such that at each modulus maxima $(s, l)$ in the cone define by (1) we have $\left|w_{s, l}\right| \leq A 2^{s \alpha}$

The Lipschitz regularity is computed by finding coefficient $\alpha$ such that $A 2^{s \alpha}$ approximates at best the decay of the wavelet coefficients over a range of scales larger than one.

Approaches $[1,4]$ use the Lipschitz property that near sharp edges, the wavelet coefficients decay exponentially over scale. At each index, an exponential fit over scale was attempted for the wavelet coefficients. In [1] if the fit was close enough to the exponential then it was used to predict the detail signal at the finer scale. If not, nothing was added to the data. In [4] the approach is similar to [1], but it tries to extrapolate features in textured regions as well. Since the Lipschitz condition does not work as well in the textured regions, the authors identify two other constraints that the higher resolution image must obey. Their algorithm alternatively projects the signal to satisfy the three basic constraints. In both cases the two directions in the image were treated independently using $1 \mathrm{D}$ predictions over scale.

\section{ADDING DETAIL}

The approach to image interpolation which we call 'Prediction of Image Detail' can be explained with the help of Figure 1. In Figure 1 the high resolution image is represented as the signal $X$ at the input to the filter bank. We assume that the low resolution, more coarsely sampled image is the result of a low-pass filtering operation followed by decimation to give the signal $A$. The low-pass filter, $L$, represents the effects of the image acquisition system. If we were able

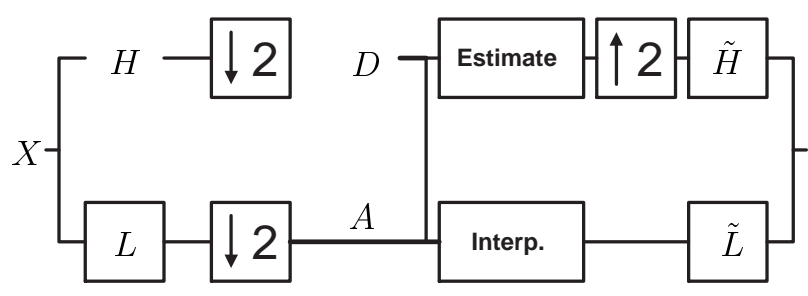

Fig. 1. Problem Formulation
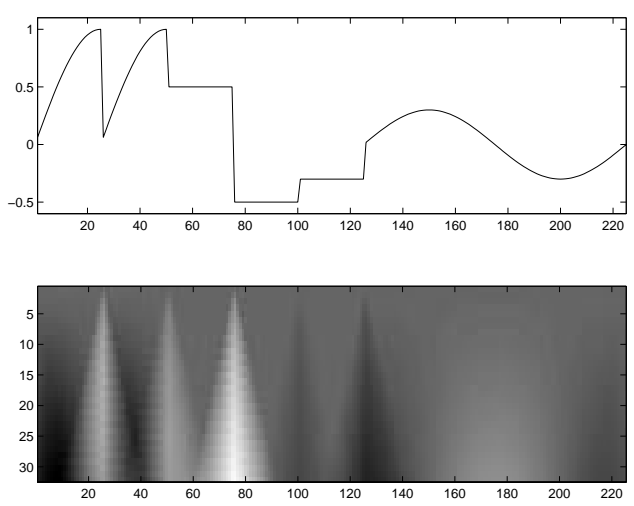

Fig. 2. Cone of Influence: Signal and Wavelet Transform

to filter the original high-resolution signal with the high pass filter $H$ to obtain the detail signal $D$ in Figure 1, and if we had a perfect reconstruction filter bank, it would then be possible to reconstruct the original image. We do not have access to the detail signal $D$, however, so we must estimate or predict what it must be.

Our approach to adding image detail is based on the behavior of edges across scales in the scale-space domain. To gain an understanding of our approach, in Fig. 1 we have generated a signal $f$ and ploted its dyadic decomposition in the scale-space domain. The approach of $[1,4]$ was to use only the modulus maxima information to estimate the detail coefficients at finest level. However Fig. 2 suggests that there may be a lot more to adding details, than just using the modulus maxima information. It suggests, that maybe we can use the entire cone of influence, from the coarser scales, for adding details to the finest scale.

In particular, the energy of the wavelet coefficients around edges, is concentrated inside the cone of influence. Or, equivalently, the energy outside the cone of influence is small. We use this observation together with the theory of optimal recovery for estimating the coefficients of the fine scale from the known coefficients, inside the cone of influence, at the coarser scales. 


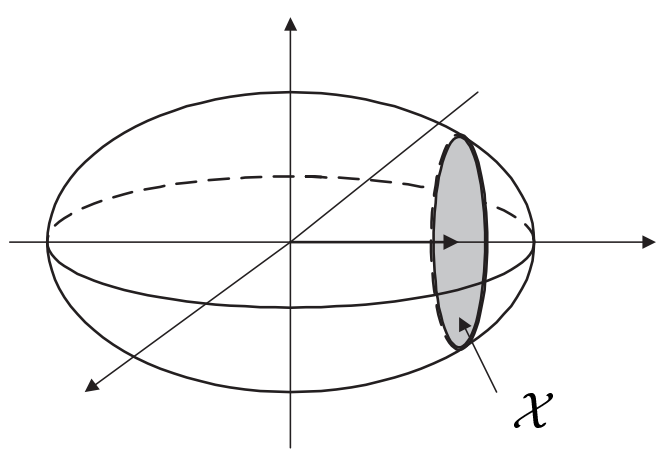

Fig. 3. Geometric Diagram of Ellipsoid Class

\section{OPTIMAL RECOVERY}

In this section we briefly review the theory of optimal recovery as applied to the interpolation problem [9]. We then propose an applications, based on a wavelet decomposition image model, of this theory to image interpolation. The interpolation problem may be viewed as a problem of estimating missing samples of a signal. This latter problem can be examined using the theory of optimal recovery. The theory of optimal recovery provides a broader setting, which not only illuminates some of the standard results on interpolation, but also indicates how to analyze the interpolation problem when the signal inputs belong to filter classes, other quadratic, ellipsoidal classes, or other types of classes. Locally, at location $n_{0}$, we model the image as belonging to a certain ellipsoid signal class $K$ based on the wavelet coefficients:

$$
\begin{aligned}
K & =\left\{f \in R^{n}: \operatorname{Energy}\left(w_{\text {outside of cone }}\right) \leq 1\right\} \\
& =\left\{f \in R^{n}: f^{T} \mathbf{Q} f \leq 1\right\} \\
& =\left\{f \in \mathbf{R}^{n}: f^{T} \mathbf{I I}^{T} f \leq 1\right\}
\end{aligned}
$$

where $\mathbf{Q}=\mathbf{I I}^{T}$ must be chosen to represent the energy of the wavelet coefficients outside the cone of influence. That is, $\mathbf{I}$ is the collection of $\psi_{s, l}$ such that $n_{0}$ is not included in the support of $\psi_{s, l}$. The filter class $K$ is represented by the ellipsoid of Fig. 3.

The known samples, in the cone of influence, determine a hyper-plane $\mathcal{X}$. The intersection of the hyper-plane and ellipsoid is a hyper-circle in $\mathcal{X}$. The intersection depends upon the known wavelet coefficients inside the cone of influence, $w_{\text {cone }}$ : we call it $C\left(w_{\text {cone }}\right)$. Formally,

$$
C\left(w_{\text {cone }}\right)=\left\{f \in \mathcal{X}: \mathbf{I} f=w_{\text {cone }},\|f\|_{Q} \leq 1\right\}
$$

For a linear mapping $\mathbf{U}$, the image of $C\left(w_{\text {cone }}\right)$ under $\mathbf{U}$ is the range of values that $\mathbf{U} f$ can take. The optimal recovery problem is to select the value in $\mathcal{X}$ which is a best approximation over all $\mathbf{U} f$ in $\mathbf{U} C\left(w_{\text {cone }}\right)$. The Chebyshev center has been shown to be the minimum $Q$-norm signal on the hyper-plane determined by the known samples. The solution to this problem is well-known: see Golomb and Weinberger [9]. If the collection of known samples is $w_{\text {cone }}$, the minimum norm signal is $\mathbf{I}^{-1} w_{\text {cone }}$, where $\mathbf{I}^{-1}$ is the pseudo-inverse with respect to the $Q$-norm. The signal $\mathbf{I}^{-1} w_{\text {cone }}$ is the unique signal in $\mathcal{X}$ with the property,

$$
\left\|\mathbf{I}^{-1} w_{\text {cone }}\right\|_{Q}=\inf _{\mathbf{I} f=w_{\text {cone }}}\|f\|_{Q}
$$

In addition to the optimal estimate $\hat{f}\left(n_{0}\right)$ for the pixel to be interpolated, $f\left(n_{0}\right)$, this theory also provides an expression for the remaining worst-case error. The worst case error is the point furthest from the center of the hyper-circle in the direction of the estimate we are making. When the best approximation is used, this maximum error is given by

$$
\begin{aligned}
E\left(\text { error }_{\text {max }}\right) & =\max _{C\left(w_{\text {cone }}\right)}\left|f\left(n_{0}\right)-\hat{f}\left(n_{0}\right)\right| \\
& =\sqrt{1-\left\|\mathbf{I}^{-1} w_{\text {cone }}\right\|_{Q}^{2}}\left\|P_{F} \phi_{n_{0}}\right\|_{Q},
\end{aligned}
$$

where $P_{F} \phi_{n_{0}}$ is the orthogonal projection of the $f\left(n_{0}\right)$ representer (the vector which by taking the $Q$ inner product with $f$, picks up the $n_{0}^{t h}$ entry of $f$ ), onto the plane parallel to the hyper-plane $\mathcal{X}$.

Note that the only part of the error term which depends on the values of the known wavelet coefficients is $\left\|\mathbf{I}^{-1} w_{\text {cone }}\right\|_{Q}$. This can be viewed as a measure of how consistent the data samples are with the signal class model, a class membership measure. It was used used as a weight of deciding how much of the predicted detail should be used. The weight is larger around edges.

\section{RESULTS}

Using the prediction over scale algorithm, we have used Daubechies number 2 wavelets to low pass filter the original image (Fig. 4) and then decimate by two to obtain Fig. 5. (Note that Fig. 5 is the decimated image, enlarged using pixel replication.) In [5] it was shown that the optimal interpolation filter for a prefiltered (with linear filter $h$ ) and down-sampled image, in the Golomb-Weinberger sense, is the autocorrelation function of $h$. In our example, we have filtered the down-sampled image with the autocorrelation of the decimation filter (which turns out to be cubic interpolation) to obtain the cubic interpolated image of Fig. 6. Finally, using the optimal recovery approach presented in this paper we have added detail coefficients to obtain the image of Fig. 7

\section{CONCLUSION}

In this paper we have presented a method of adding detail based on the cone of influence and the theory of optimal re- 


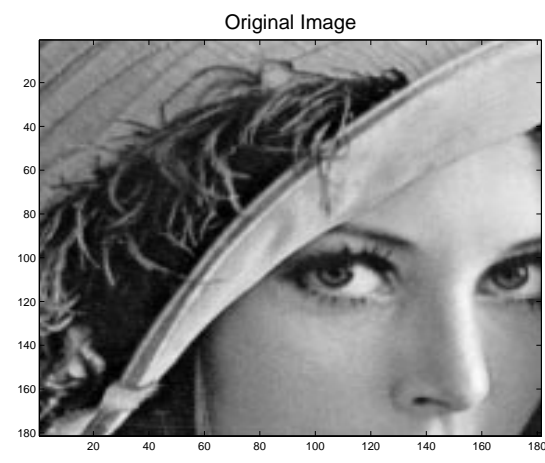

Fig. 4. Original Image

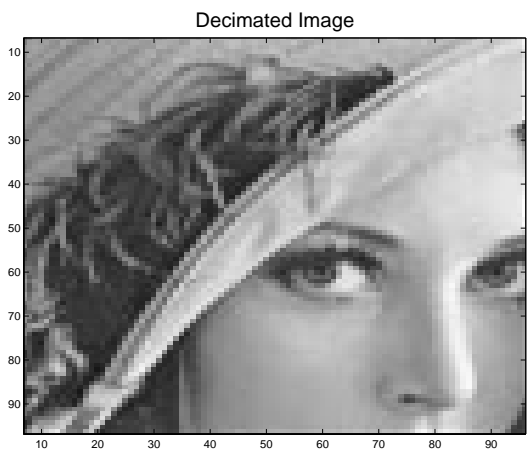

Fig. 5. Decimated Image

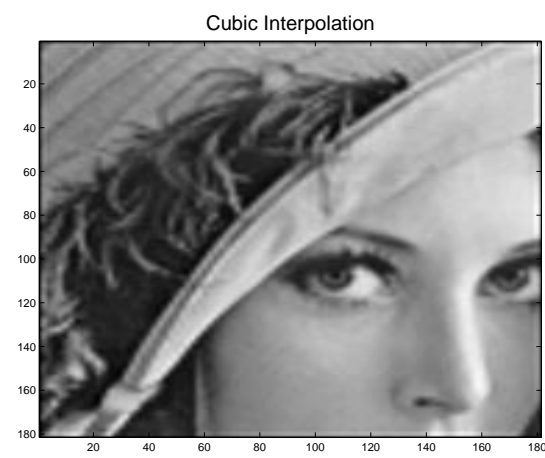

Fig. 6. Cubic Interpolation

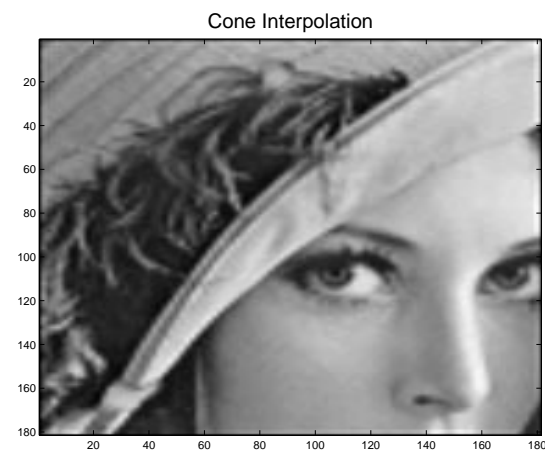

Fig. 7. Cone Interpolation covery. If the down-sampled image was assumed to have originated from a low-pass process, followed by decimation, good results were obtained by estimating the fine scale wavelet coefficients. The estimate of the fine scale detail was based on the cone of influence and optimal recovery theory.

\section{REFERENCES}

[1] W. Knox Carey, Daniel B. Chuang and Sheila S. Hemami, "Regularity-Preserving Image Interpolation," IEEE Transactions on Image Processing, Vol. 8, No. 9 (Sept. 1999), 1293-1297.

[2] M. S. Crouse, R. D. Nowak, and R. G. Baraniuk, "Wavelet-Based Signal Processing Using Hidden Markov Models," IEEE Transactions on Signal Processing (Special Issue on Wavelets and Filterbanks), April 1998.

[3] Stephane Mallat, "A Wavelet Tour of Signal Processing," Academic Press, 1998.

[4] S. Grace Chang, Zoran Cvetkovic, and Martin Vetterli, "Resolution Enhancement of Images Using Wavelet Transform Extrema Interpolation," IEEE ICASSP, (May 8-12, 1995), pp. 2379-2382.

[5] Ram G. Shenoy and Thomas W. Parks, "An Optimal Recovery Approach to Interpolation," IEEE Transactions on Signal Processing, Vol. 40, No. 8, (August 1992), pp. 1987-1992.

[6] Stephane Mallat and Sifen Zhong, "Characterization of Signals from Multiscale Edges," IEEE Transactions on Pattern Analysis and Machine Intelligence, Vol. 14, No. 7 (July. 1992), pp. 710-732.

[7] Stephane Mallat and Wen Liang Hwang, " Singularity Detection and Processing with Wavelets," IEEE Transactions on Information Theory, Vol. 38, No. 2 (March. 1992), pp. 617-643.

[8] Jean Babaud, Andrew P. Witkin, Michael Baudin, and Ricard O. Duda, "Uniqueness of the Gaussian Kernel for Scale-Space Filtering," IEEE Transactions on Pattern Analysis and Machine Intelligence, Vol. 8, No. 1 (Jan. 1986), pp. 26-33.

[9] M. Golomb and H. F. Weinberger, "Optimal approximation and Error Bounds," On Numerical Approximation, R. E. Langer ed., The University of Wisconsin Press, Maddison, pp. 117-190, 1959. 